\title{
Article \\ The Role of Autosensitivity Control (ASC) in Cochlear Implant Recipients
}

\author{
Federica Di Berardino ${ }^{1,2} \mathbb{D}$, Diego Zanetti ${ }^{1,2, * \mathbb{C}}$, Daniela Soi ${ }^{3}$, Lara Dalla Costa ${ }^{3}$ and Sandro Burdo ${ }^{3}$ \\ 1 Department of Specialist Surgical Sciences, Audiology Unit, Fondazione IRCCS Ca' Granda Ospedale \\ Maggiore Policlinico, 20122 Milan, Italy; federica.diberardino@unimi.it \\ 2 Department of Clinical Sciences and Community Health, University of Milan, 20122 Milano, Italy \\ 3 Italian Association "Liberi di Sentire", formerly Director (SB) and Assistant (LDC) at Audiovestibology Unit \\ Varese Hospital, 21100 Varese, Italy; danisoi@yahoo.it (D.S.); laradallacosta@me.com (L.D.C.); \\ sanburdo@tin.it (S.B.) \\ * Correspondence: diego.zanetti@policlinico.mi.it; Tel.: +39-0255033901; Fax: +39-0250320754
}

\section{check for} updates

Citation: Di Berardino, F.; Zanetti, D.; Soi, D.; Costa, L.D.; Burdo, S. The Role of Autosensitivity Control (ASC) in Cochlear Implant Recipients. Audiol. Res. 2021, 11, 22-30. https:// doi.org/10.3390/audiolres11010003

Received: 30 December 2020

Accepted: 12 January 2021

Published: 21 January 2021

Publisher's Note: MDPI stays neutral with regard to jurisdictional claims in published maps and institutional affiliations.

Copyright: (c) 2021 by the authors. Licensee MDPI, Basel, Switzerland. This article is an open access article distributed under the terms and conditions of the Creative Commons Attribution (CC BY) license (https:// creativecommons.org/licenses/by/ $4.0 /)$.
Abstract: The purpose of the study was to examine the subjective and objective potential advantage for speech understanding in noise achieved by cochlear implant (CI) recipients when using the autosensitivity control (ASC) input signal processing in combination with the adaptive dynamic range optimization (ADRO). Eighteen subjects ( 8 females, 10 males, mean age $17.7 \pm 6.7$ ) were enrolled in a prospective open blinded comparative study between the ASC + ADRO condition vs. the ADRO alone; 16 were sequential binaural and 2 were monoaural CI recipients. All patients had been wearing their CI for at least 3 years, had no additional disabilities, had an age-appropriate receptive and expressive language. Word recognition performances in noise (at signal-to-noise ratio $+5 \mathrm{~dB}$ HL) were significantly better in the ADRO-alone condition than in the ADRO + ASC condition. $(p=0.03)$ These objective outcomes were in agreement with the subjective reports. No significant difference was found in quiet. Our results, apparently in contrast with other reports in the literature, suggest that the decision of adding the slow-acting automatic reduction in microphone sensitivity provided by ASC should be limited to selected CI recipients.

Keywords: ADRO; ASC; CI; cochlear implantation; word recognition score; speech in noise; fitting

\section{Introduction}

Among the input signal pre-processing systems of cochlear implantations (CI), many authors have described the benefits of the autosensitivity control (ASC) [1-6]. The ASC was firstly introduced in the body-worn mini sound processor in 1989 by Cochlear Limited (Lane Cove, Australia) [7] and was designed to improve speech recognition by keeping the speech level in the comfortable loudness region in the presence of background noise [8]. It is a slow-acting linear compression pre-processing system that acts after the amplification performed by the speech-processor microphone; its purpose is to optimize the speechto-noise ratio, based on the unmodulated noise input ("noise floor") of the surrounding environment [9]. The ASC attack time is generally 2 second, and the release time is 8 second; its long release time is used to reduce distortion and/or maintain a comfortable listening level [10]. While the overall noise floor is continuously monitored by the slow dynamics of ASC [8], any transient and abrupt increase in noise is not controlled by a slow system that misses them completely [9]. As a result, every time an unexpectedly loud sound occurs in the environment, the user perceives an uncomfortably loud stimulation $[11,12]$. In order to reduce the effect of impulsive noises, another pre-processing automatic gain control (AGC) has been implemented, called AGC-input, working together with ASC in a system defined as "dual-loop AGC". While ASC acts like a slow-acting AGC, the AGC-input is built to react quickly in response to sudden loud noise, by reducing the gain of high-amplitude 
inputs and avoiding distortion and peak clipping $[13,14]$. Modulation rates and depths are used to classify a signal as speech (i.e., $2-20 \mathrm{~Hz}$ ) or noise.

The ASC is activated when the average noise floor increases exceeding the automatic gain kneepoint, defined as the AutoSensitivity Break-Point (ASBP), that is, $57 \mathrm{~dB}$ SPL at default settings [15]. Once the ambient noise floor reaches or exceeds $57 \mathrm{~dB}$ SPL, whether it is impulsive or not, the sensitivity of the speech-processor microphone is slowly reduced by the ASC according to the level of the background noise; this produces a subjective reduction of loudness. In order to keep the noise floor at least 10-15 dB SPL below the AGC kneepoint [16], the ASC shifts all the instantaneous input dynamic range (IIDR), with a compression at a ratio of 1:1. Since the ASC acts as a linear amplifier with minimal variations in the signal, it reduces the whole input gain and not only the high intensities or the unmodulated sounds (Figure 1). This input signal pre-processing systems have been implemented in the Smartsound ${ }^{\circledR}$ software by Cochlear (Cochlear Limited, Sydney, Australia); however, the literature on the real-world joint benefit of AGCs to ASC in CI recipients is still scarce, and some authors have raised the hypothesis that the ASC might negatively affect the interaural level difference cues [6]. In our clinical practice, we noticed that most of our patients frequently reported to prefer to use only the Adaptive Dynamic Range Optimization (ADRO) alone rather than ASC + ADRO in noisy conditions. The aim of this study was to examine the subjective and objective potential benefits or disadvantages in speech recognition in noise by the use of ASC input signal processing.
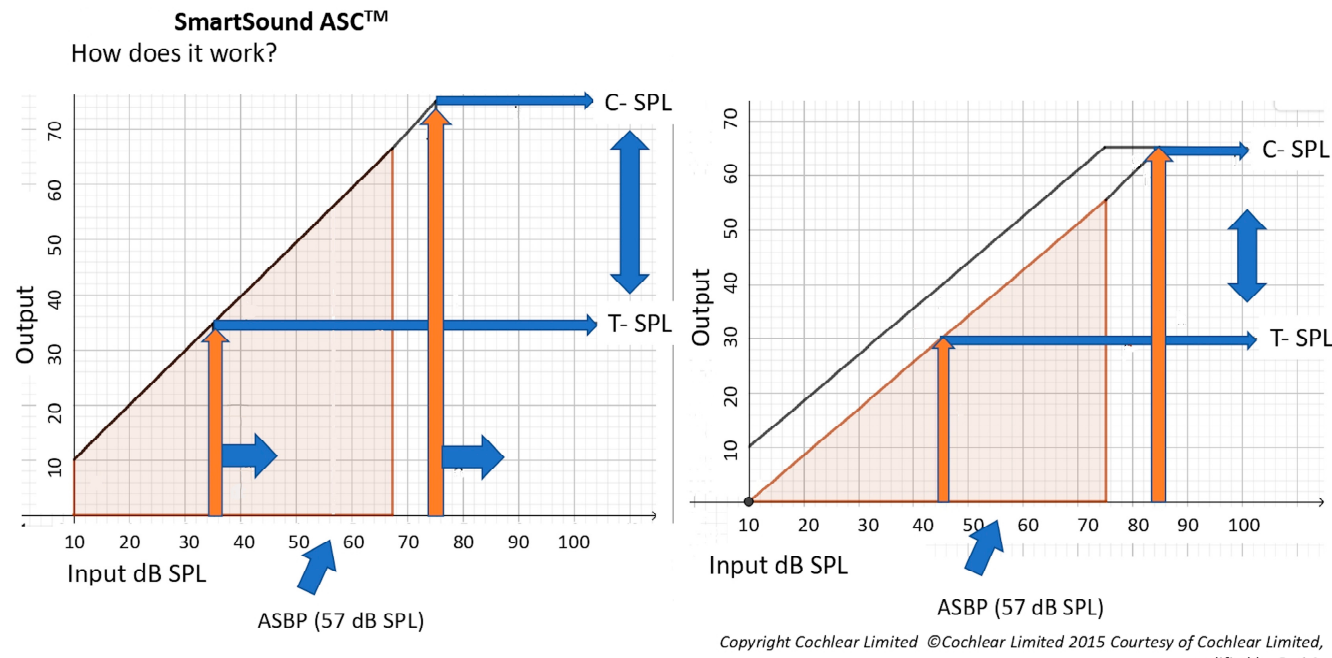

Figure 1. The autosensitivity control (ASC) input-output curves.

\section{Materials and Methods}

The study design is prospective, cross-sectional, observational blinded, focused on the direct effect of ASC vs. ADRO. As in the study conducted with children by Rakszawski B. et al. [17], the other preprocessing systems (Whisper ${ }^{\circledR}$, Beam $^{\circledR}$ and Zoom ${ }^{\circledR}$ ) (Cochlear Limited, Sydney, Australia) that modify the microphone directionality and adjust the electrode gain in noise were deactivated.

Eighteen CI recipients ( 8 females and 10 males, mean age of $17.7 \pm 6.7$ years, ranging from 10 to 46 years) were randomly selected to be enrolled in the study. All subjects had age-appropriate receptive and expressive language; none had additional disabilities.

The demographics and the main clinical features of the patients are reported in Table 1. 
Table 1. CI recipients demographic informations.

\begin{tabular}{|c|c|c|c|c|c|c|c|c|c|}
\hline Participants & Age at Test & Sex & $\begin{array}{c}\text { Device } \\
\text { Configuration }\end{array}$ & $\begin{array}{c}\text { Implanted Ear } \\
\text { (Left(Right/Both) }\end{array}$ & $\begin{array}{c}\text { Type of } \\
\text { Hearing Loss }\end{array}$ & $\begin{array}{c}\text { Age at 1st CI } \\
\text { (Years of CI Use) }\end{array}$ & $\begin{array}{c}\text { Age at 2nd } \\
\text { CI }\end{array}$ & $\begin{array}{c}\text { Interaural } \\
\text { Equivalence }\end{array}$ & Internal Device \\
\hline 1 & 16 & M & Bilateral & B & congenital & 3 & 12 & Yes & CI24RE; CI24RE \\
\hline 2 & 13 & M & Bimodal & $\mathrm{L}$ & congenital & 2 & & & CI24RE \\
\hline 4 & 10 & $\mathrm{~F}$ & Bilateral & B & congenital & 1 & 8 & Yes & $\mathrm{CI} 24 \mathrm{RE} ; \mathrm{CI} 24 \mathrm{RE}$ \\
\hline 5 & 30 & $\mathrm{~F}$ & Bilateral & B & congenital & 21 & 8 & No & CI24RE; CI512 \\
\hline 6 & 12 & $\mathrm{~F}$ & Bilateral & B & congenital & 1 & 10 & Yes & $\mathrm{CI} 24 \mathrm{RE} ; \mathrm{CI} 24 \mathrm{RE}$ \\
\hline 7 & 12 & $\mathrm{~F}$ & Bilateral & B & acquired & 8 & 3 & Yes & CI24RE; CI24RE \\
\hline 9 & 47 & M & Bilateral & B & acquired & 38 & 9 & Yes & CI24RE; CI512 \\
\hline 10 & 8 & M & Bilateral & B & congenital & 1 & 7 & Yes & CI24RE; CI24RE \\
\hline 11 & 8 & $\mathrm{M}$ & Bilateral & B & congenital & 1 & 7 & yes & CI24RE; CI24RE \\
\hline 12 & 31 & $\mathrm{~F}$ & Bilateral & B & congenital & 18 & 13 & No & CI24RE; CI24RE \\
\hline 13 & 18 & $\mathrm{~F}$ & Bimodal & $\mathrm{R}$ & acquired & 16 & & & CI512 \\
\hline 14 & 14 & $\mathrm{M}$ & Bilateral & B & congenital & 4 & 10 & yes & CI24RE; CI24RE \\
\hline 15 & 14 & $\mathrm{~F}$ & Bilateral & $\mathrm{B}$ & congenital & 2 & 12 & No & CI24RE; CI512 \\
\hline 16 & 14 & M & Bilateral & B & congenital & 1 & 13 & Yes & CI24RE; CI512 \\
\hline 17 & 10 & M & Bilateral & B & congenital & 1 & 9 & yes & CI24RE; CI24RE \\
\hline
\end{tabular}

$\mathrm{M}$ = males; $\mathrm{F}$ = female; $\mathrm{R}$ = right ear; L: left ear; $\mathrm{B}$ = both ear; CI24RE: Receiver-Stimulator Contour Advance. 
Participants had been implanted either with the Nucleus Freedom ${ }^{\circledR}$ (Cochlear Limited, Sydney, Australia) or with a Nucleus 5 (CI512) ${ }^{\circledR}$ (Cochlear Limited, Sydney, Australia). Sixteen were sequential binaural CI and two were monaural CI recipients. All patients used a CP810 ${ }^{\circledR}$ (Cochlear Limited, Sydney, Australia) speech processor. The mean duration of $\mathrm{CI}$ use was 9 years, with a range from 2 to 13 years. All participants used the Advanced Combination Encoder (ACE) speech coding strategy.

All CI recipients' maps were checked and re-programmed weekly for 6 to 8 weeks. Minimum stimulation (T) levels were set at counted thresholds to ensure audibility, and maximum stimulation (C) levels were set at loud but comfortable levels to ensure the dynamic range was maximized. All participants in this study were programmed according to this protocol after at least 3 years from their initial CI activation. All 22 electrode contacts were active in all $\mathrm{CI}$ recipients. A monopolar stimulation was selected for all patients, and stimuli were trains of symmetric biphasic pulses of $25 \mathrm{msec}$ duration; the duration of each pulse-train was $600 \mathrm{msec}$. The rate of stimulation was 2400 pulses per second per channel (pps/ch) in $70 \%$ of patients and $1200 \mathrm{pps} / \mathrm{ch}$ in the other $30 \%$.

All participants were fitted with two maps: one map included ADRO and the other map included ADRO + ASC; the patients were blinded to the allocation of the 2 maps and were asked to use arbitrarily the two maps in everyday life and in the different environment conditions (e.g., at home, in the pub, in the street traffic, at work). The sensitivity and volume of the microphone were kept at default settings. All subjects should indicate the map they considered most favourable and were interviewed regarding the subjective qualitative differences between the two maps.

All subjects underwent a speech-tracking (ST) test in noise [18] by a trained audiologist in a double-blind setting, since both the patient and the Audiologist were unaware of which maps were tested. The ST takes four minutes to be accomplished; it is routinely used in the fitting protocol to evaluate the effectiveness of hearing aids and CI, given its high sensitivity in detecting any decline in hearing performance in intra-subject testing. It also investigates the recognition of ongoing speech, and it can be used as a method of training [19]. According to Burdo et al. [20], by counting the number of words repeated correctly in a minute, the ST returns a word recognition score (WRS) in a free-running connected discourse.

The speech material consisted in 20 sentences extracted from common written texts chosen on the basis of the patient's age; for adults, it consists of reading a newspaper $($ level +3$)$, delivered live-voice by a professional reader (always the same one in all sessions with all patients), without lip-reading, in quiet and in noise, at a root mean square level of $65 \mathrm{~dB}$ (A) SPL, located $1 \mathrm{~m}$ directly in front of the proband ( $0^{\circ}$ azimuth). The background ("cocktail party") noise was delivered from a second loudspeaker located $1 \mathrm{~m}$ behind the patient's shoulders ( $180^{\circ}$ azimuth). Both signal and noise were adjusted in order to achieve a constant signal-to-noise (SNR) ratio of $+5 \mathrm{~dB}$ HL. [21] The speech tests were performed in a sound-treated booth; live voice output was constantly monitored during the test, and the loudspeaker output was checked with a sound meter before each patient's session.

Statistical analysis. The Kolmogorov-Smirnov test was used to assess the normality of the examined variables. The paired Student's t-test was carried out to determine the statistical significance of average differences of scores obtained with the two maps. A $p$-value of less than 0.05 was considered statistically significant. All statistics were calculated using the Statistical Package for the Social Sciences 24.0 for Windows software package (SPSS Inc., Chicago, IL, USA).

\section{Results}

No association was found between the outcomes obtained with the two maps (with and without ASC) and the patients' characteristics. WRS in quiet were not statistically different between ASC + ADRO compared to ADRO alone.

Conversely, when the speech tracking task was performed with a masking noise, the difference between ADRO + ASC and ADRO alone was significant: 15 out of 18 patients 
(83.34\%) scored significantly better at WRS with the ADRO alone, as shown in Figure 2, $(p=0.03)$ with an average improvement of 7 words per minute at a SNR of $+5 \mathrm{~dB}$ HL. Patient 10 had the same WRS at SNR $+5 \mathrm{~dB}$ HL with the two maps; only patient 11 and 12 had a slightly higher number of correct answers at WRS with ADRO + ASC compared with ADRO alone. However, they both indicated the ADRO as the preferred map, complaining about the reduction of speech recognition in noise with ASC, due to a subjective significant lowering of the speech signal intensity in noise.

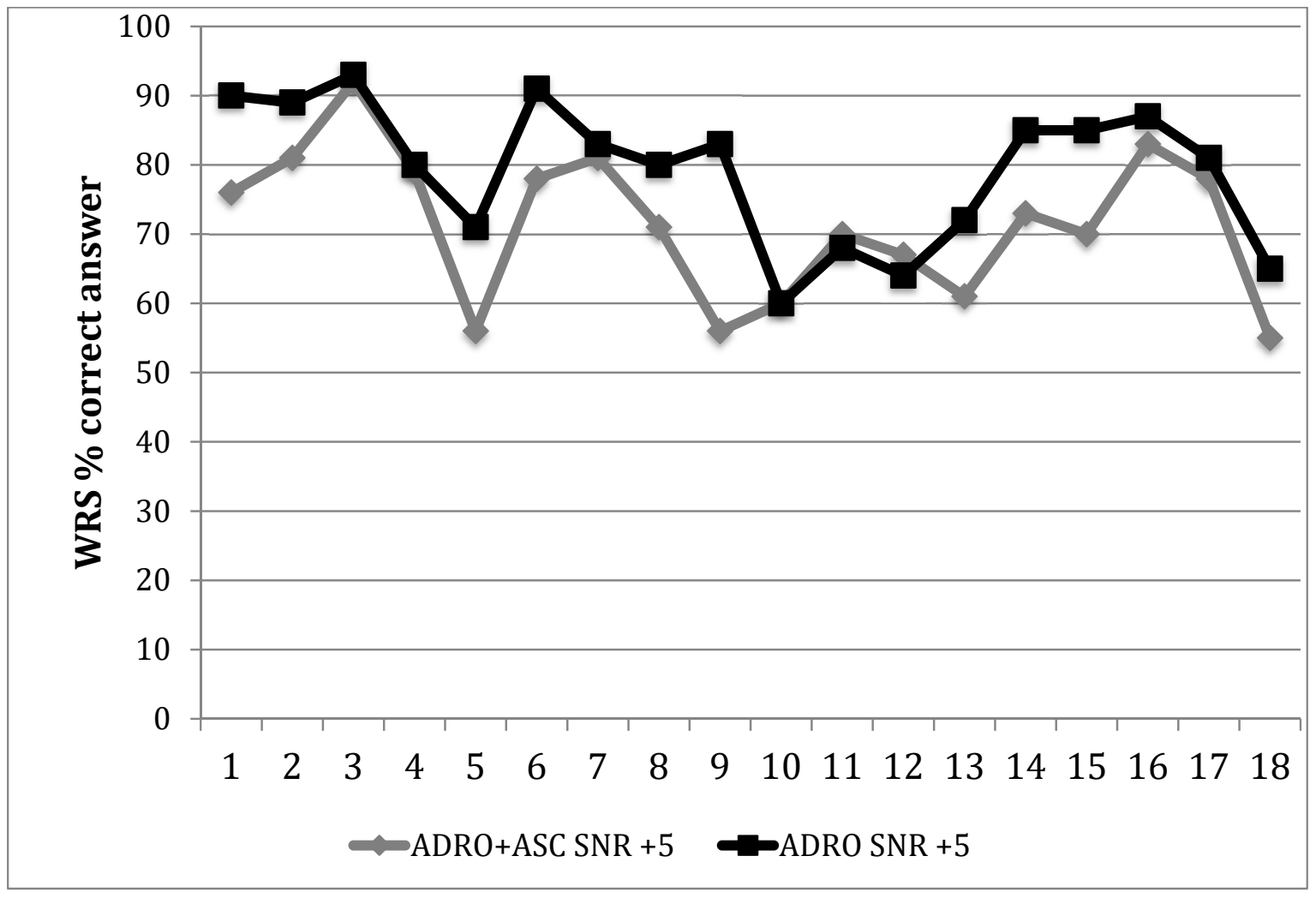

Figure 2. Word recognition scores for each CI recipient with the two settings.

From a subjective viewpoint, all patients reported discomfort when using the map with ADRO + ASC in quiet and noisy environments. Only two CI recipients reported to perceive sometimes a clearer voice with the ASC map in noise but, nevertheless, they preferred and used more frequently the ADRO alone. The major complaints with the ASC + ADRO map concerned the lowering of the loudness of speech and the perceived fluctuations of the voice, which resulted in a significant subjective reduction of speech comprehension in noise. On the contrary, all patients reported a certain improvement of the SNR in a noisy environment with ADRO alone.

\section{Discussion}

In the literature, the ASC has been consistently reported to provide a 2-3 dB SNR improvement of the $50 \%$ correct answers threshold either alone or in combination with ADRO [22]. In this current open, double-blinded investigation, we failed to observe the expected objective improvement in noise with ASC. Our results indicate that ADRO alone allows a significantly better response, with an average improvement of 7 words per minute at an SNR of $+5 \mathrm{~dB}$ HL. The patients' feedback was also in agreement with these objective results, since all the patients reported preferring the map with ADRO alone in different noisy environments. The major complaints reported by the patients when using ASC resemble the disadvantages of the slow-acting compression systems applied in hearing aids [23]: 
- Loudness perception that is not restored to "normal". The output level typically shifts only slightly from the input level; it may be difficult for the user to judge the strength of sound sources. This may have adverse effects on the interpretation of environmental sounds [24].

- When the acoustic scene changes abruptly, such as when two voices with markedly different levels alternate or when switching rapidly from a loud to a quiet environment (e.g., when leaving a noisy room), the gain takes a second or two to reach the value appropriate for the new situation. Hence, the aid may appear to become "dead" for a while.

- When trying to listen to one (target) voice in the presence of another (background) voice, a normally hearing person can extract information about the target during the temporal dips in the background, a process called "Listening in the dips" [25]. The information in the dips may be at a relatively low level, especially when the mean target level is lower than the mean background level. Hearing-impaired people have a reduced ability to listen in the dips [23], partly because of reduced audibility of the target speech in the dips [26]. A slow-acting system may be of limited benefit in this situation because the gain does not increase significantly during brief dips in the input signal; the gain applied during the dips is essentially the same as the gain applied during the peaks in the input.

Conversely, as already reported by James et al. in 2003 [27], the results in quiet observed in this study did not show any difference between the two maps. Therefore, we tried to understand why introducing the ASC did not bring in our data the expected objective improvement in noise.

First of all, according to the classification of the compression algorithms proposed by Dillon [12], the ASC has a linear slow-acting dynamics with a medium compression threshold. While fast-acting compression with a short release time ( $<50 \mathrm{~ms})$ such as the AGC-input component is designed to follow the intensity variations encountered at the phonemic or syllabic level of speech, longer release time ( $>200 \mathrm{~ms})$, such as the ASC, is used to reduce distortion and/or maintain a comfortable listening level; as a result, the ASC is more useful when the change in gain is needed for larger intensity levels and longer duration. For this reason, the improvement observed with ASC may differ in the presence of other types of noise or environments (i.e., steady-state noise or diffuse noise in a reverberant environment) [2], and different levels of speech and noise influence the real-life outcomes.

As an example, a child's everyday listening environment is much noisier than that encountered by the typical adult [28]. However, we noticed that the school background noise reported in the ASC literature is much higher (dB Leq/day: $87.3 \mathrm{dBA}$ to $95.5 \mathrm{dBA}$ [29], with a mean of $56 \mathrm{dBA}$ during silent classroom reading to $73 \mathrm{~dB}$ A during group activities [28]) than the noise level found in our schools, which are more similar but lower than other European reports (dB Leq/day $51.5 \mathrm{dBA} \pm 4.5 \mathrm{dBA}$; ranging from 38 to $58 \mathrm{dBA}$ ) [30]. This observation might confirm that the beneficial effect of ASC + ADRO is more evident in diagnostic and real-life settings in which there are higher levels of constant surrounding noise and higher root-mean-squared output level.

As far as CIs are concerned, previous research demonstrated that loudness grows as an exponential function of the current intensity [31,32]. It is also known that the loudness growth functions are dependent on pulse rate, with loudness growing faster at low pulse rates $[33,34]$. Electrical thresholds and maximum acceptable loudness levels, in fact, vary for stimulation rates between 250 and $2400 \mathrm{pps} / \mathrm{ch}$ on the absolute current level (CL) value [35]. T and $C$ levels decrease as a function of pulse rate but the slopes of the $C$ level vs. pulse rate functions are shallower than the slopes of the $\mathrm{T}$ level vs. pulse rate functions. This ends up in a larger dynamic range at low pulse rates compared to that at high pulse rates [36]. The sensitivity setting determines when the AGC will start acting and is aligned to C-level stimulation [37]. 
Another source of discrepancy in the research studies is represented by the range of stimulation rates employed in different CI: it varies extensively from low $(<500 \mathrm{pps} / \mathrm{ch})$ to moderate (500-1000 pps /ch) to high (>1000 pps/ch) [37]. Comparing the methods used in this study to the others reported in the literature in which the beneficial effects of ASC was very evident, we applied higher stimulation rates and lower $C$ levels.

As already mentioned, ASC works like an "automatic input volume control compressor", modifying the T and C levels, as reported in Figure 1. Furthermore, the ASC is considered a broadband strategy, since the manipulation of microphone sensitivity affects the entire possible spectral range from 188 to $7938 \mathrm{~Hz}$ [28]. Several studies have investigated the effect of increasing the compression ratio and shortening the compression time constants on subjectively perceived sound quality, but the parameter of compression channel bandwidth (or number of compression channels) has not received much attention in the literature [38]. Therefore, mapping information (e.g., T/C levels, IDR/IID, number of active electrodes and rate of stimulation) was generally not cited in initial studies and shows large variations in more recent reports might significantly affect the results. In the present study, $\mathrm{C}$ levels were set at a lower level and at a higher rate of stimulation than those of other previous studies; thus, the addition of ASC might have led to a significant reduction of loudness as described by our patients. This, in turn, would explain the significant hearing threshold decline that negatively affects the CI recipient's clinical performance; a similar effect is observed when a microphone fails, causing a persistent reduction in the sound processor sensitivity.

Objective measures for detecting $\mathrm{C}$ levels, such as those based on the stapedial reflexes [39], might provide more uniform data to evaluate the effect of these pre-processing compressions.

\section{Conclusions}

Our data indicate that word recognition performances in noise of experienced CI recipients were significantly better in the ADRO-alone than in the ADRO + ASC condition $(p=0.03)$, both with objective measures and by subjective reports. The apparent contrast with other reports in the literature may lie in the different parameters of the electrical stimulation in the different trials.

Every CI recipient differs in T and C levels; similarly, each patient selects different preferred microphone sensitivity, volume control, and noise-reduction settings.

We wish to stress the importance of reporting all these settings' data e.g., T/C levels, IDR/ IID, number of active electrodes and rate of stimulation in order to compare the results obtained by different research groups studying Smartsound ${ }^{\circledR}$ (Cochlear Limited, Sydney, Australia) technologies.

\section{Summary of Evidence}

1. Among this study's subjects, $83.34 \%$ of patients performed significantly better with the ADRO alone rather than with ADRO + ASC.

2. The ASC is more useful when the change in gain is needed for larger intensity levels and longer duration.

3. The sensitivity setting determines when the AGC will start acting and is aligned to C-level stimulation. Mapping parameters (e.g., T/C levels, IDR/ IID, number of active electrodes and rate of stimulation) might significantly affect the results.

Author Contributions: Conceptualization, S.B.; formal analysis, investigation, resources, and data handling, L.D.C. and D.S.; writing-original draft preparation, F.D.B., S.B. and D.S.; writing-review, F.D.B.; editing and supervision, D.Z. All authors have read and agreed to the published version of the manuscript.

Funding: This research received no external funding. 
Institutional Review Board Statement: In The study was conducted according to the guidelines of the Declaration of Helsinki and approved by the Institutional Review Board (or Ethics Committee) of Fondazione IRCCS Ca' Granda Ospedale Maggiore Policlinico of Milan, Italy (protocol code 468, 2 Gennaio 2019).

Informed Consent Statement: Informed consent was obtained from all subjects involved in the study.

Data Availability Statement: The data presented in this study are available on request from the corresponding author. The data are not publicly available due to privacy restrictions.

Acknowledgments: We wish to thank the cochlear implant recipients who participated in this study; Cochlear Limited, Sydney, Australia for technical informations and for Figure 1 release; Sara Cavicchiolo and Enrico Luigi Fagnani for their helpful technical hints.

Conflicts of Interest: The authors declare no conflict of interest. There are no funding sources; no external influences had a role in the design of the study, in the collection, analyses, or interpretation of data, in the writing of the manuscript, or in the decision to publish the results.

\section{References}

1. Wolfe, J.; Schafer, E.C.; Heldner, B.; Mülder, H.; Ward, E.; Vincent, B. Evaluation of Speech Recognition in Noise with Cochlear Implants and Dynamic FM. J. Am. Acad. Audiol. 2009, 20, 409-421. [CrossRef] [PubMed]

2. Wolfe, J.; Schafer, E.C.; John, A.; Hudson, M. The Effect of Front-End Processing on Cochlear Implant Performance of Children. Otol. Neurotol. 2011, 32, 533-538. [CrossRef] [PubMed]

3. Gifford, R.H.; Revit, L.J. Speech Perception for Adult Cochlear Implant Recipients in a Realistic Background Noise: Effectiveness of Preprocessing Strategies and External Options for Improving Speech Recognition in Noise. J. Am. Acad. Audiol. 2010, 21, 441-451. [CrossRef] [PubMed]

4. Brockmeyer, A.M.; Potts, L.G. Evaluation of Different Signal Processing Options in Unilateral and Bilateral Cochlear Freedom Implant Recipients Using R-Space ${ }^{\mathrm{TM}}$ Background Noise. J. Am. Acad. Audiol. 2011, 22, 065-080. [CrossRef] [PubMed]

5. Hersbach, A.A.; Arora, K.; Mauger, S.J.; Dawson, P.W. Combining directional microphone and single-channel noise reduction algorithms: A clinical evaluation in difficult listening conditions with cochlear implant users. Ear Hear. 2012, 33, e13-e23. [CrossRef] [PubMed]

6. Potts, L.G.; Kolb, K.A. Effect of Different Signal-Processing Options on Speech-in-Noise Recognition for Cochlear Implant Recipients with the Cochlear CP810 Speech Processor. J. Am. Acad. Audiol. 2014, 25, 367-379. [CrossRef] [PubMed]

7. Cochlear Limited Dual omni-directional microphone technology. Cochlear N34480F ISS1 2010 May 10th. Sydney, Australia. Available online: https://www.cochlear.com/au/en/home/products-and-accessories/cochlear-nucleus-system/nucleus-soundprocessors (accessed on 3 December 2020).

8. Spahr, A.J.; Dorman, M.F.; Loiselle, L.H. Performance of Patients Using Different Cochlear Implant Systems: Effects of Input Dynamic Range. Ear Hear. 2007, 28, 260-275. [CrossRef]

9. Seligman, P.; Whitford, L. Adjustment of appropriate signal levels in the Spectra 22 and mini speech processors. Ann Otol. Rhinol. Laryngol. Suppl. 1995, 166, 172-175.

10. Walker, G.; Dillon, H. Compression in Hearing Aids: An Analysis, a Review and Some Recommendations; National Acoustic Laboratories Report No. 90; Australian Government Publishing Service: Canberra, Australia, 1982.

11. Khing, P.P.; Swanson, B.A.; Ambikairajah, E. The Effect of Automatic Gain Control Structure and Release Time on Cochlear Implant Speech Intelligibility. PLoS ONE 2013, 8, e82263. [CrossRef]

12. Dillon, H. Compression systems in hearing aids. In Dillon H. Hearing Aid; Thieme Medical Publishers: New York, NY, USA, 2012; Chapter 6; pp. 170-197.

13. Stöbich, B.; Zierhofer, C.M.; Hochmair, E.S. Influence of Automatic Gain Control Parameter Settings on Speech Understanding of Cochlear Implant Users Employing the Continuous Interleaved Sampling Strategy. Ear Hear. 1999, 20, 104-116. [CrossRef]

14. Boyle, P.J.; Büchner, A.; Stone, M.A.; Lenarz, T.; Moore, B.C.J. Comparison of dual-time-constant and fast-acting automatic gain control (AGC) systems in cochlear implants. Int. J. Audiol. 2009, 48, 211-221. [CrossRef] [PubMed]

15. Patrick, J.F.; Busby, P.A.; Gibson, P.J. The Development of the Nucleus ${ }^{\circledR}$ Freedom ${ }^{\mathrm{TM}}$ Cochlear Implant System. Trends Amplif. 2006, 10, 175-200. [CrossRef] [PubMed]

16. Braida, L.D.; Durlach, N.I.; De Gennaro, S.V.; Peterson, P.M.; Bustamante, D.K. Hearing aids: A review of past research on linear amplification, amplitude compression, and frequency lowering. ASHA Monogr. 1979, $19,1$.

17. Rakszawski, B.; Wright, R.; Cadieux, J.H.; Davidson, L.S.; Brenner, C. The Effects of Preprocessing Strategies for Pediatric Cochlear Implant Recipients. J. Am. Acad. Audiol. 2016, 27, 85-102. [CrossRef]

18. De Filippo, C.L.; Scott, B. A method for training and evaluating the reception of ongoing speech. J. Acoust. Soc. Am. 1978, 63, 1186-1192. [CrossRef] [PubMed]

19. Razza, S.; Burdo, S. An underestimated issue: Unsuspected decrease of sound processor microphone sensitivity, technical, and clinical evaluation. Cochlea-Implants Int. 2011, 12, 114-123. [CrossRef] [PubMed] 
20. Burdo, S.; Poggia, C.; Giuria, P. TAUV (Test Abilità Uditive Varese). I Care 1995, 2 (Suppl. 1), 3-36.

21. Burdo, S. Impianto cocleare binaurale. In Cuda D. Impianti Cocleari; Italy, TorGraf Edition; Quaderni Monografici di Aggiornamento A.O.O.I.: Galatina, Italy, 1997; Chapter 7; p. 139. Available online: https://www.aooi.it/contents/attachment/c4 /impiantiCocleari.pdf (accessed on 11 November 2020).

22. Moore, B.C.J. The Choice of Compression Speed in Hearing Aids: Theoretical and Practical Considerations and the Role of Individual Differences. Trends Amplif. 2008, 12, 103-112. [CrossRef] [PubMed]

23. Peters, R.W.; Moore, B.C.J.; Baer, T. Speech reception thresholds in noise with and without spectral and temporal dips for hearing-impaired and normally hearing people. J. Acoust. Soc. Am. 1998, 103, 577-587. [CrossRef]

24. Gatehouse, S.; Noble, W. The Speech, Spatial and Qualities of Hearing Scale (SSQ). Int. J. Audiol. 2004, 43, 85-99. [CrossRef]

25. Duquesnoy, A.J. Effect of a single interfering noise or speech source on the binaural sentence intelligibility of aged persons. $J$. Acoust. Soc. Am. 1983, 74, 739-743. [CrossRef] [PubMed]

26. Bacon, S.P.; Opie, J.M.; Montoya, D.Y. The Effects of Hearing Loss and Noise Masking on the Masking Release for Speech in Temporally Complex Backgrounds. J. Speech Lang. Hear. Res. 1998, 41, 549-563. [CrossRef] [PubMed]

27. James, C.J.; Skinner, M.W.; Martin, L.F.A.; Holden, L.K.; Galvin, K.L.; Holden, T.A.; Whitford, L. An Investigation of Input Level Range for the Nucleus 24 Cochlear Implant System: Speech Perception Performance, Program Preference, and Loudness Comfort Ratings. Ear Hear. 2003, 24, 157-174. [CrossRef] [PubMed]

28. Gifford, R.H.; Olund, A.P.; DeJong, M. Improving Speech Perception in Noise for Children with Cochlear Implants. J. Am. Acad. Audiol. 2011, 22, 623-632. [CrossRef] [PubMed]

29. Clark, W.W.; Govett, S.B. School-related noise exposure in children. In Proceedings of the Association for Research in Otolaryngology Mid-Winter Meeting, St. Petersburg, FL, USA, 8 February 1995.

30. Pujol, S.; Levain, J.-P.; Houot, H.; Petit, R.; Berthillier, M.; Defrance, J.; Lardiès, J.; Masselot, C.; Mauny, F. Association between Ambient Noise Exposure and School Performance of Children Living in An Urban Area: A Cross-Sectional Population-Based Study. J. Hered. 2013, 91, 256-271. [CrossRef] [PubMed]

31. Shannon, R.V. Threshold and loudness functions for pulsatile stimulation of cochlear implants. Hear. Res. 1985, 18, 135-143. [CrossRef]

32. Chatterjeec, M.; Fu, Q.-J.; Shannon, R.V. Effects of phase duration and electrode separation on loudness growth in cochlear implant listeners. J. Acoust. Soc. Am. 2000, 107, 1637-1644. [CrossRef]

33. Zeng, F.-G.; Galvin, J.J. Amplitude Mapping and Phoneme Recognition in Cochlear Implant Listeners. Ear Hear. 1999, 20 , 60-74. [CrossRef]

34. Galvin, J.J.; Fu, Q.-J. Influence of stimulation rate and loudness growth on modulation detection and intensity discrimination in cochlear implant users. Hear. Res. 2009, 250, 46-54. [CrossRef]

35. Skinner, M.W.; Holden, L.K.; Holden, T.A.; Demorestt, M.E. Effect of Stimulation Rate on Cochlear Implant Recipients' Thresholds and Maximum Acceptable Loudness Levels. J. Am. Acad. Audiol. 2000, 11, 203-213.

36. Zhou, N.; Xu, L.; Pfingst, B.E. Characteristics of detection thresholds and maximum comfortable loudness levels as a function of pulse rate in human cochlear implant users. Hear. Res. 2012, 284, 25-32. [CrossRef] [PubMed]

37. Arora, K. Cochlear Implant Stimulation Rates and Speech Perception. In Modern Speech Recognition Approaches with Case Studies; IntechOpen: Rijeka, Croatia, 2012.

38. Ole Hau Hanne Pernille Andersen Hearing Aid Compression: Effects of Speed, Ratio and Channel Bandwidth on Perceived Sound Quality. Available online: http://www.audiologyonline.com/articles/hearing-aid-compression-effects-speed-770 (accessed on 3 December 2020).

39. Guida, M.; Falcioni, M.; Di Lella, F.; Negri, M.; Fagnani, E.; Vincenti, V. Multi-electrode sequential versus single electrode stimulation to elicit the stapedial reflex during cochlear implantation: Correlation with maximum comfort level. Eur. Ann. Otorhinolaryngol. Head Neck Dis. 2019, 136, 169-172. [CrossRef] [PubMed] 